\title{
Diffuse alveolar hemorrhage
}

\author{
Deepa Panikkath MD, Swetha Gadwala MD, Brooke Mills BS, Ragesh Panikkath MD
}

\begin{abstract}
Diffuse alveolar hemorrhage $(D A H)$ is a rare life threatening condition characterized by bleeding into the alveolar spaces. Although it classically presents as hemoptysis, anemia, diffuse alveolar infiltrates, and acute respiratory failure, it is often a diagnostic and therapeutic challenge for clinicians. It is associated with both immune and nonimmune causes. Prompt recognition and treatment of this entity are crucial. This article reviews the common etiologies, diagnosis, and management of the patients with $D A H$.
\end{abstract}

Key words: Diffuse alveolar hemorrhage, autoimmune, vasculitis, infiltrates, anemia

\section{INTRODUCTION}

Diffuse alveolar hemorrhage (DAH) is a clinical syndrome characterized by widespread bleeding arising from the pulmonary microvasculature. ${ }^{1}$ It was first described by Osler in $1904 .^{2}$ DAH can be a manifestation of either a systemic disease or localized injury to the lung. Most patients present with symptoms of cough, dyspnea, hemoptysis, and the presence of new alveolar infiltrates. Early bronchoscopy with diagnostic lavage is necessary for diagnosis. Therapy is mainly supportive with a combination of corticosteroids and immunosuppressive drugs.

\section{Pathophysiology}

The key event in DAH is disruption of the alveolar capillary basement membrane caused either by inflammation or injury to the arterioles, venules,

Corresponding author: Deepa Panikkath MD

Contact Information: Deepa.Panikkath@ttuhsc.edu DOI: 10.12746/swrccc 2015.0309.114 or capillaries. ${ }^{3}$ It can be caused by autoantibodies to the alveolar capillary endothelium, or antibodies to the alveolar basement membrane, or direct alveolar injury. Commonly three patterns of DAH are seen: pulmonary capillaritis, bland hemorrhage, and diffuse alveolar damage.

1. Pulmonary capillaritis is the most common pattern. ${ }^{4}$ It is characterized by neutrophilic infiltration into the alveolar septae, endothelial edema, injury, and fibrinoid necrosis. This leads to loss of structural integrity and bleeding into alveolar spaces. ${ }^{1,4}$

2. Bland hemorrhage is not associated with inflammation or destruction of the alveolar capillaries, venules, and arterioles but with widespread leaking of RBCs into the alveoli. ${ }^{1}$

3. Diffuse alveolar damage is characterized by interstitial and intra-alveolar edema, capillary congestion, microthrombi, and hyaline membrane formation. ${ }^{1}$

\section{Clinical pRESENTATION}

$\mathrm{DAH}$ can occur at any age and is sometimes the initial manifestation of the underlying disease. It can be acute, subacute, or repetitive with variable severi- 
ty. It is commonly associated with antibasement membrane antibodies (anti-GBM) disease, connective tissue diseases, and systemic vasculitides. Nonimmune causes include drugs, toxins, stem cell transplantation, infections, and valvular heart disease. Common etiologies are usually grouped based on the pattern of involvement and are listed in Table 1.1,4

Table 1 Differantial diagnosis of diffuse alveolar hemmorrhage

\section{A. Disorders associated with pulmonary capillaritis Systemic vasculitides \\ Granulomatosis with polyangiitis, Churg Strauss syndrome \\ Microscopic polyangiitis (MPA) \\ Isolated pauci-immune pulmonary capillaritis \\ Connective tissue disorders \\ SLE, RA, MCTD*, scleroderma \\ Immune complex mediated \\ Goodpasture's syndrome, Henoch Schonlein purpura, Ig A nephropathy}

Others

Hematopoietic stem cell transplantation

Behçet syndrome

B. Disorders associated with diffuse alveolar damage

Acute respiratory distress syndrome

Cytotoxic drugs, radiation therapy

SLE

Crack cocaine inhalation

C. Disorders associated with bland pulmonary hemorrhage

Drugs - anticoagulants, antiplatelet, thrombolytics, diphenylhydantoin, amiodarone, penicillamine, PTU

DIC*

SLE

Mitral stenosis, mitral regurgitation

Infections - HIV, infective endocarditis

SLE-systemic lupus erythematosus, RA-rheumatoid arthritis, MCTD-mixed connective tissue disease, PTU-propylthiouracil, DIC-disseminated intravascular coagulation

The classic triad consists of anemia, pulmonary infiltrates on chest x-ray, and hemoptysis. ${ }^{5,6}$ However, DAH can present in about $40 \%$ of the cases without hemoptysis even during severe bleeding into alveolar spaces..$^{4,6}$ New infiltrates on chest radiographs ac- companied by a fall in hemoglobin are more sensitive indicators of this condition. This clinical presentation can be confused with bacterial and opportunistic infections when patients present with dyspnea, cough, chest pain, tachypnea, and fever. The physical examination may be quite nonspecific. Nasopharyngeal, cutaneous, or ocular signs suggest a connective tissue or vasculitis. The presence of palpable purpuric skin lesions, acute glomerulonephritis, mononeuritis multiplex, nasal septal erosion suggests a vasculitis. In a study of 97 patients with $\mathrm{DAH}$, four parameters suggested immune-related DAH: onset of respiratory symptoms $\geq 11$ days, fatigue and/or weight loss during the month prior to presentation, arthralgia or arthritis, and proteinuria $\geq 1 \mathrm{~g} / \mathrm{L} .{ }^{7}$ Vasculitis, mainly granulomatosis with polyangiitis, was the most common cause of DAH in a retrospective study. ${ }^{8}$ SLE is the most common connective tissue diseases associated with $\mathrm{DAH}$, and most cases are associated with lupus nephritis. ${ }^{2}$ The prevalence ranges from $2 \%$ to $5 \%$ of patients with SLE. ${ }^{9}$ It is the presenting symptom in $5-10 \%$ of patients with Goodpasture's syndrome. ${ }^{10}$ Male gender and smoking are risk factors for $\mathrm{DAH}$ in this diagnosis.

\section{Diagnosis}

The diagnosis of DAH requires clinical recognition and supporting clinical, laboratory, radiologic, and pathologic information. The key elements in the approach to this disorder include establishing the diagnosis of $\mathrm{DAH}$ and identifying the underlying cause (Figure 1). ${ }^{31}$ Symptoms in conjunction with bloody bronchoalveolar lavage can establish the diagnosis of $\mathrm{DAH}$.

\section{History and physical examination}

A thorough history should be taken since $\mathrm{DAH}$ can be secondary to various immune mediated disorders, vasculitides, infections, valvular disorders, bone marrow transplantation, etc. A detailed drug and occupation history should be obtained. Physical examination findings are usually nonspecific. They can include signs associated with an underlying systemic vasculitis, such as rash, purpura, eye lesions, and hepatosplenomegaly. ${ }^{11,12}$ 


\section{Laboratory assessment}

Laboratory evaluation typically reveals acute or chronic anemia, leukocytosis, elevated erythrocyte sedimentation rates, and elevated C-reactive protein levels. Comprehensive laboratory studies should include cultures to exclude infection, complete blood counts, and a complete metabolic panel. Urine drug screens should be performed. Renal abnormalities can occur in Goodpasture's syndrome and granulomatosis with polyangiitis. If a pulmonary-renal syndrome is suspected due to hematuria or renal impairment, ANCA levels should be checked. C-ANCA positivity is most consistent with granulomatosis with polyangiitis and P-ANCA with antimyeloperoxidase specificity favors the diagnosis of MPA or Churg-Strauss syndrome. The presence of anti-GBM antibodies in serum is diagnostic of Goodpasture's syndrome. If lupus or antiphospholipid antibody syndrome is suspected, complement fractions $\mathrm{C} 3$ and $\mathrm{C} 4$, anti-double stranded DNA, and antiphospholipid antibodies levels are needed. ${ }^{12,13}$

\section{Imaging studies}

New or old patchy or diffuse alveolar infiltrates are often seen on radiographs. Kerley $B$ lines indicate the possibility of mitral valve disease. Reticular interstitial opacities with minimal to absent honeycombing can be seen in cases with recurrent hemorrhage causing pulmonary fibrosis. Computed tomography especially high-resolution studies will show areas of consolidation interspersed with ground-glass opacities and preserved normal alveolar spaces (Figure 2). Additional features seen that may suggest systemic disorders include cavitating pulmonary nodules and masses caused by granulomatosis with polyangiitis, fibrosis and bronchiectasis in MPA, and pleural effusions in SLE. ${ }^{3}$ Nuclear imaging has limited role in evaluating $\mathrm{DAH} .{ }^{12,13}$

\section{Pulmonary function tests}

Pulmonary function tests and arterial blood gases are essential since DAH can cause impairment of oxygen transfer and subsequent hypoxemia. The diffusing capacity of carbon monoxide (DLCO) may be increased since the blood in the lungs binds inhaled carbon monoxide. A serial increase in DLCO can indicate progressive hemorrhage, but this test may be impractical given the instability of patients with $\mathrm{DAH} .{ }^{12}$ Pulmonary function tests reveal restrictive physiology with a decreased total lung capacity and a decreased forced vital capacity with a preserved ratio of forced expiratory volume in 1 second to the forced vital capacity. Obstructive defects are seen less often in these patients but can occur if neutrophilic infiltration from blood extravasation into alveoli causing the release of reactive oxygen species and proteolytic enzymes also causes small airway damage. If an obstructive pattern is seen, diseases, such as microscopic polyangiitis, granulomatosis with polyangiitis, pulmonary capillaritis, or idiopathic pulmonary hemosiderosis, should be considered. ${ }^{12}$

\section{Bronchoscopy}

Bronchoscopy is the key investigation needed to diagnose alveolar hemorrhage by lavage and to exclude other associated infections. ${ }^{14}$ Bronchoscopy has higher yield if performed within the first 48 hours. Persistent or increasing blood on three sequential lavage aliquots from one affected area of lung supports the diagnosis of alveolar hemorrhage, but DAH must be suspected before this procedure is considered. In cases of subacute or recurrent DAH, the number of hemosiderin-laden macrophages should be counted. ${ }^{12,14}$ In addition, bronchoalveolar lavage specimens should be sent for routine bacterial, mycobacterial, fungal, and viral cultures, and Pneumocystis stains. Transbronchial biopsy is of limited used in the diagnosis of DAH as the area of involvement is often patchy. However, it can be used when alternative diagnoses, such as sarcoidosis, are being considered. ${ }^{12}$

\section{Histology}

The three common patterns seen in DAH are: pulmonary capillaritis, bland alveolar hemorrhage, and diffuse alveolar damage (Figure 3). Various systemic disorders and medications associated with these patterns are listed in Table 1. Pulmonary capillaritis is the most common type. ${ }^{4}$ Tissue biopsies of kidney, skin, 
or nasal mucosa are often preferred over open lung biopsy to establish the underlying systemic disease since they are less invasive. Linear IgG deposition along capillary basement membrane is diagnostic of
Goodpasture's syndrome. Marked granular immune complex deposition can be seen in connective tissue disorders like SLE; IgA deposits suggest Henoch Schonlein purpura or $\lg$ A nephropathy.

Figure 1 Diagnostic algorithm

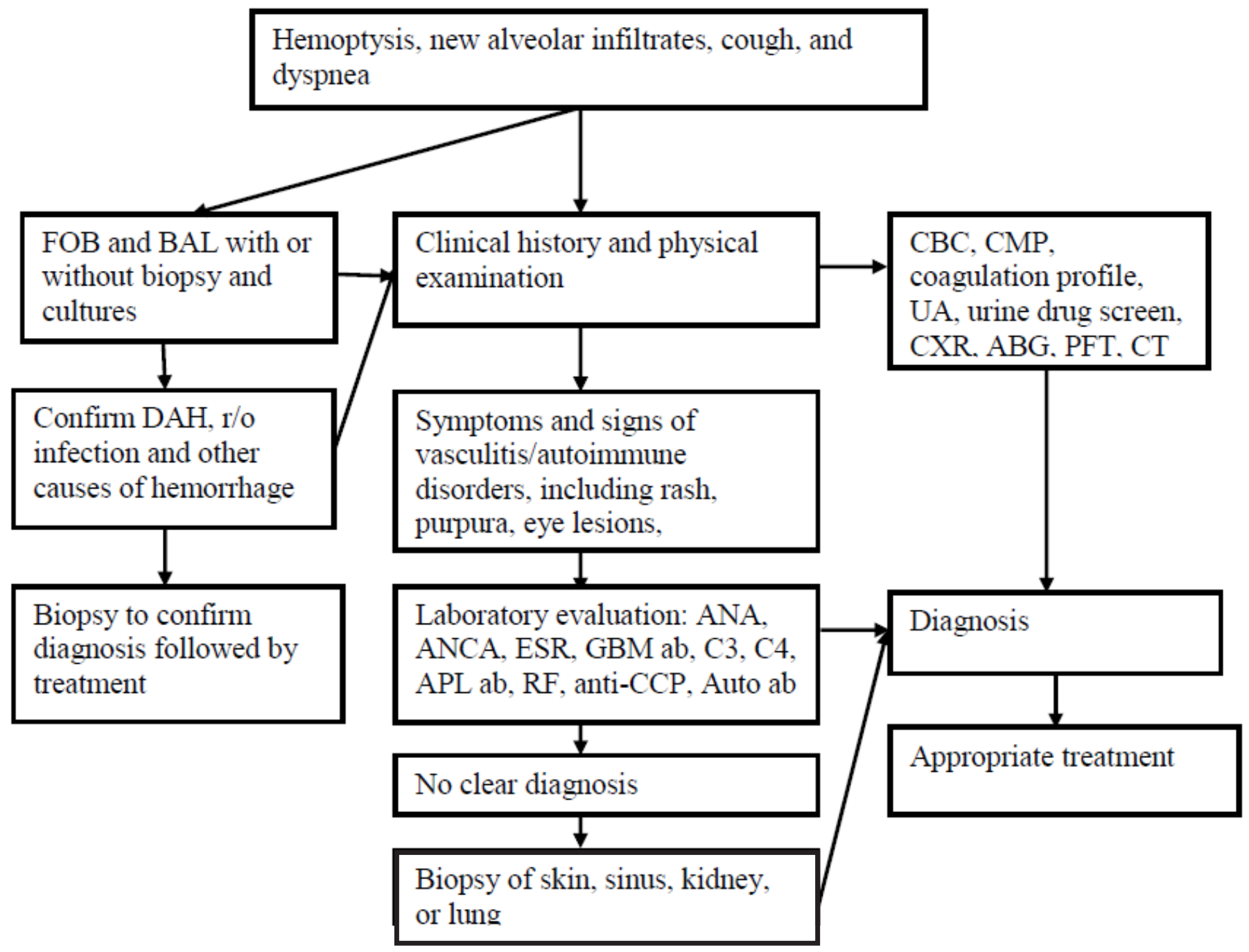

Figure 1: FOB: fiberoptic bronchoscopy, BAL: Bronchoalveolar lavage, ANA: anti-nuclear antibody, ANCA: anti-neutrophil cytoplasmic antibodies, ESR: erythrocyte sedimentation rate, GBM: glomerular basement membrane, APL: anti-phospholipid, ab: antibody, RF: Rheumatoid factor, anti-CCP: anti-cyclic citrullinated peptide, auto ab: auto antibody. UA: urinalysis, CMP: comprehensive metabolic profile, CXR: chest X-ray, PFT: pulmonary function test. Adapted from Park MS, 2013. ${ }^{13}$ 

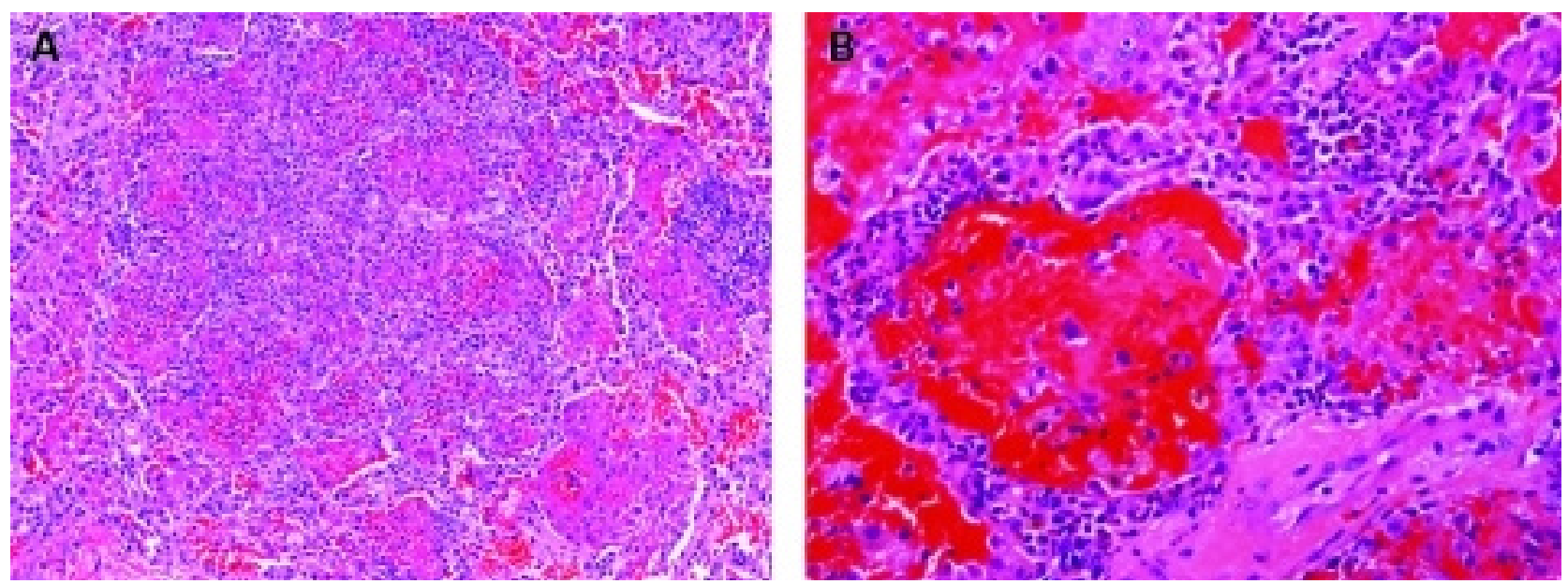

Figure 2: Biopsy specimen showing alveolar spaces filled with blood that lines alveolar surfaces to form hyaline membrane. Image from Leslie KO, $2009^{15}$ Used under creative commons license: http://creativecommons.org/licenses/by/2.0/.

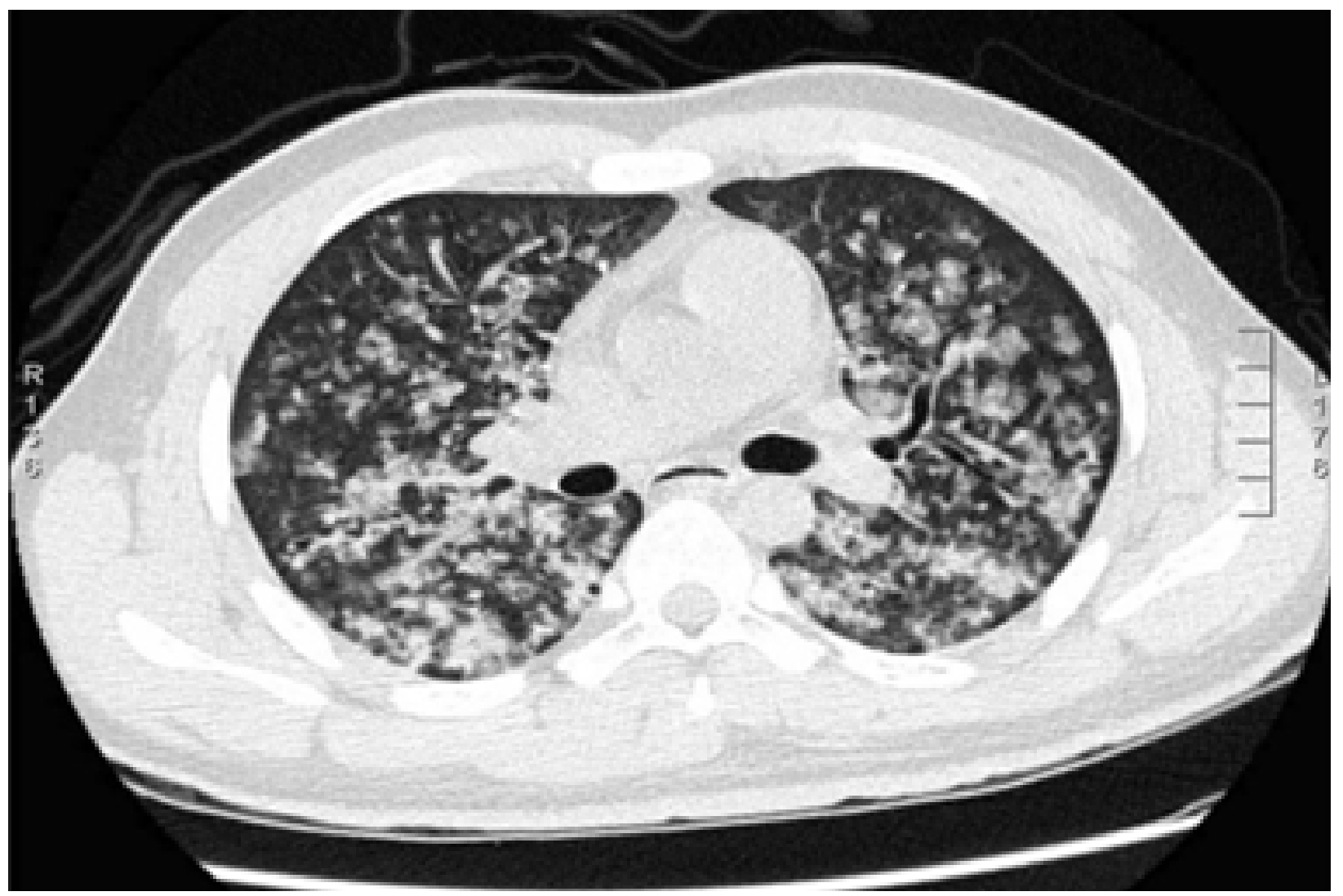

Figure 3: CT Thorax showing bilateral alveolar infiltrates and ground glass opacities. Image from Kim JP, Park JJ, Kim NJ, et al. ${ }^{16}$ Used under creative commons license: http://creativecommons. org/licenses/by-nc/3.0/ 


\section{Management}

Management of DAH includes stabilization of the patient's respiratory status and rapid correction of the underlying disease or cause. Adequate ventilation and perfusion status should be established with oxygen, mechanical ventilation as needed, and correction of coagulation abnormalities. Commonly accepted goals for correction are a platelet count $>50,000 / \mu \mathrm{L}$ and an INR $<1.5 .{ }^{17}$ Empiric antibiotic therapy is often started since it is difficult to exclude underlying infection and since many of these patients are on immunosuppressive drugs for immune mediated diseases. During ventilation, use of lung protective strategies with tidal volumes of $6 \mathrm{ml} / \mathrm{kg}$ and inspiratory plateau pressures below $30 \mathrm{cmH}_{2} \mathrm{O}$ can help avoid increasing damage to pulmonary microvasculature. Treatment of a few specific disorders associated with $\mathrm{DAH}$ is discussed below.

\section{ANCA associated vasculitis}

$\mathrm{DAH}$ is one of the most serious complications of ANCA-associated vasculitis. Therapy is divided into aggressive induction and maintenance phases. Standard induction regimens include intravenous methylprednisolone (500-1,000 mg) for 3-5 days. As clinical improvement occurs, the dose can be reduced and weaned down over several weeks to a maintenance dose once remission is attained. Glucocorticoid therapy is combined with cytotoxic agents. Cyclophosphamide is the preferred agent at a dose $15 \mathrm{mg} /$ $\mathrm{kg}$ administered intravenously as a pulse every 2-3 weeks initially or $1-2 \mathrm{mg} / \mathrm{kg} /$ day orally. ${ }^{18,19}$ Rituximab has been found to be noninferior to cyclophosphamide as an induction agent. ${ }^{19}$ Plasma exchange may be useful in refractory cases during the early stage, but its exact benefit is unclear. ${ }^{18,21}$ One observational study has shown it to reduced mortality by $50 \%$. Rarely ECMO has been used when conventional mechanical ventilation fails. This allows a reduction in ventilatory settings ( $\mathrm{FiO}_{2}$ and pressure) and prevents further lung damage. ${ }^{22}$ IVIG has been used in recurrent or persistent disease, but the response is often transient. ${ }^{23}$ Following successful induction patients are on a maintenance regimen for at least 24 months or longer. Methotrexate weekly (10-20 mg) and azathioprine daily $(2 \mathrm{mg} / \mathrm{kg}$ ) are well studied treatment options..$^{24,25}$

\section{MPA}

Treatment, as with ANCA-associated vasculitis, with glucocorticoids, cyclophosphamide, and plasmapheresis is the recommended regimen for induction of remission. ${ }^{4}$ Recombinant factor VIla has been used to treat severe DAH in MPA in patients with refractory disease. ${ }^{24,26}$

\section{SLE}

Corticosteroids and immunosuppressive drugs are the cornerstones of treatment. High dose IV corticosteroids with methylprednisolone $(1 \mathrm{~g}$ daily for 3 days followed by 1 to $2 \mathrm{mg} / \mathrm{kg} / \mathrm{d}$ in divided doses orally) are recommended initially. This is followed by gradual taper to maintenance doses of oral corticosteroids. Cyclophosphamide is the most commonly used immunosuppressive agent, either as daily oral dose (2-5 mg/kg/day or monthly IV pulse doses. ${ }^{4,26}$ Alternative agents include azathioprine, rituximab, or intravenous y globulin. In refractory cases, plasmapheresis has been used; however, there is no benefit in survival rates. There is theoretical concern for increased autoantibody synthesis as a rebound phenomenon while using plasmapheresis. ${ }^{4}$ ECMO has been used as rescue therapy in severe refractory cases of DAH complicating SLE. ${ }^{28}$

\section{Goodpasture's syndrome}

In patients without renal involvement there is often a good response to corticosteroids alone, but in presence of renal failure, immunosuppressive drugs and plasmapheresis are essential. 5,29 The extent and duration of plasmapheresis are not known, but 4-liter plasma exchanges daily or every other day are usually performed. Plasmapheresis is continued for 2-3 weeks or until the patient's clinical course has improved and serum anti-GBM antibodies are not de- 
tected. ${ }^{24}$ Immunosuppressive therapy with steroids and cyclophosphamide is required to inhibit antibody production and treat the rebound hypersynthesis of the antibodies that may occur following discontinuation of plasma exchange.

\section{Primary antiphospholipid syndrome (APLS)}

Combination therapy of glucocorticoids and other immunosuppressive drugs (cyclophosphamide, azathioprine, or mycophenolate mofetil) primarily combined with the intravenous administration of immunoglobulin G (IV IgG) and/or plasma exchange has been successful in treating these patients. ${ }^{30}$ They require temporary discontinuation of anticoagulation and hence are at high risk for recurrent venous and/ or arterial thrombosis.

\section{Hematopoietic SCT patients}

Standard therapy is high dose steroids. ${ }^{4}$ Aminocaproic acid has been shown to reduce mortality in $\mathrm{DAH}$ in these patients. ${ }^{31}$

\section{Drug induced DAH}

Treatment is mainly supportive with discontinuation of the offending drug and correction of coagulation abnormalities. ${ }^{5}$ Penicillamine-associated DAH has required immunosuppressive therapy and plasmapheresis. ${ }^{6}$

\section{Newer therapies}

Recombinant factor VIla (rVIla) has been used to control bleeding in refractory immune mediated causes and in non-immune causes not effectively controlled by supportive care or with treatment of the underlying disease. ${ }^{32,33}$ rVIla has been successfully administered both systemically (intravenously) or bronchoscopically. Thrombotic complications involving both arterial and venous events have been reported in some patients treated with rVIla and patients should be monitored carefully. Tranexamic acid (a synthetic antifibrinolytic drug) in either aerosolized form or as intrapulmonary injections has controlled bleeding in a few cases of $\mathrm{DAH} .{ }^{34}$

\section{OUTCOMES AND PROGNOSIS}

Long term complications include development of pulmonary fibrosis and restrictive lung disease after recurrent $\mathrm{DAH}$. The reported prognosis is generally poor, with several studies showing mortality ranging from $7 \%$ to over $50 \%$. ${ }^{32,34}$ Outcomes vary with the underlying cause; DAH associated with underlying SLE has a $50 \%$ mortality rate and can be recurrent in survivors. Poor prognostic markers include renal insufficiency, thrombocytopenia, and the need for mechanical ventilation. ${ }^{2,35}$ The mortality rate exceeds $80 \%$ in mechanically ventilated patients in ANCA associated vasculitis with low $\mathrm{PaO}_{2} / \mathrm{FIO}_{2}$ ratio $<100 \mathrm{~mm}$ $\mathrm{Hg}^{22} \mathrm{DAH}$ in SCT patients have very high mortality of $70-100 \%{ }^{36}$

\section{Conclusions}

$\mathrm{DAH}$ is a life threatening manifestation of various immune and non-immune mediated disorders. The classic presentation includes development of new alveolar infiltrates, anemia, dyspnea, and hemoptysis. Prompt identification of the underlying etiology is crucial to initiate appropriate therapy and increase chances of survival.

\footnotetext{
Author Affiliations: All authors work at Texas Tech University Health Sciences Center in Lubbock, TX. Brooke Mills is a medical student, Deepa Panikkath and Swetha Gadwala are residents in internal medicine, and Ragesh Panikkath is a fellow in cardiology.

Submitted: $12 / 10 / 2014$

Accepted: $1 / 8 / 2015$

Reviewers: Cynthia Jumper MD

Published electronically: 1/15/2015

Conflict of Interest Disclosures: None
} 


\section{REFERENCES}

1. Newsome BR, Morales JE. Diffuse alveolar hemorrhage. South Med J 2011; 104(4):269-74.

2. Zamora MR, Warner ML, Tuder R, et al. Diffuse alveolar hemorrhage and systemic lupus erythematosus. Clinical presentation, histology, survival, and outcome. Medicine (Baltimore) 1997; 76:192-202.

3. Krause ML, Cartin-Ceba R, Specks U, Peikert T. Update on diffuse alveolar hemorrhage and pulmonary vasculitis. Immunology and Allergy Clinics of North America 2012; 32(4): 587-600.

4. Lara AR, Schwarz MI. Diffuse alveolar hemorrhage. Chest 2010; 137(5):1164-71.

5. Collard HR, King T, Schwarz MI. Diffuse alveolar hemorrhage and rare infiltrative disorders of the lung. Murray and Nadel's Textbook of Respiratory Medicine, Chapter 60, 14491468 .

6. Collard HR, Schwarz MI. Diffuse alveolar hemorrhage. Clin Chest Med 2004; 25:583-592.

7. Picard C, Cadranel J, Porcher R et al. Alveolar haemorrhage in the immunocompetent host: a scale for early diagnosis of an immune cause. Respiration 2010; 80: 313-320.

8. Travis WD, Colby TV, Lombard C, Carpenter HA. A clinicopathologic study of 34 cases of diffuse pulmonary hemorrhage with lung biopsy confirmation Am J Surg Pathol 1990; 14(12):1112-1125.

9. Araujo DB, Borba EF, Silva CA, Campos LMA, Pereira RMR, Bonfa E, et al. Alveolar hemorrhage: distinct features of juvenile and adult onset systemic lupus erythematosus. Lupus 2012; 21:872-7.

10. Fontenot AP, Schwarz A, Marvin I. Diffuse alveolar hemorrhage. In: Schwarz MI, Talmadge E, eds. Interstitial lung disease. Hamilton, ON, Canada: BC Decker; 2003:632-656.

11. Albert RK. Massive Hemoptysis. In Principles of Critical Care, 3e. Hall JB, Schmidt GA, Wood LDH. 2005.

12. Ioachimescu OC. Diffuse alveolar hemorrhage: Diagnosing it and finding the cause. Cleveland Clinic J Med 2008; 4:258-280.

13. Park MS. Diffuse Alveolar Hemorrhage. Tuberculosis and Respiratory Diseases 2013; 74:151-162.

14. Dweik RA, Stoller JK. Role of bronchoscopy in massive hemoptysis. Clin Chest Med 1999; 20:89-105.

15. Leslie KO. My approach to interstitial lung disease using clinical, radiological and histopathological patterns. J. Clin. Pathol 2009. May; 62(5):387-401.

16. Kim JP, Park JJ, Kim NJ, et al. A case of diffuse alveolar hemorrhage after tonsillectomy- A case report. Korean J Anesthesiol 2012. August; 63(2):165-168.
17. Khan SA, Subla MR, Behl D, et al. Outcome of patients with small-vessel vasculitis admitted to a medical ICU. Chest 2007; 131(4):972-6.

18. Tarzi RM, Pusey CD. Current and future prospects in the management of granulomatosis with polyangiitis (Wegener's granulomatosis). Ther Clin Risk Manag 2014; 10: 279-293.

19. de Groot K, Harper L, Jayne DR, et al. Pulse versus daily oral cyclophosphamide for induction of remission in antineutrophil cytoplasmic antibody-associated vasculitis: a randomized trial. Ann Intern Med 2009; 150:670-680.

20. Keogh KA, Ytterberg SR, Fervenza FC, Carlson KA, Schroeder DR, Specks U. Rituximab for refractory Wegener's granulomatosis: report of a prospective, open-label pilot trial. Am J Respir Crit Care Med 2006; 1732:180-187.

21. Casian A, Jayne D. Plasma exchange in the treatment of Wegener's granulomatosis, microscopic polyangiitis, ChurgStrauss syndrome and renal limited vasculitis. Curr Opin Rheumatol 2011; 23(1):12-7.

22. Ahmed SH, Aziz T,Cochran J, Highland K. Use of extracorporeal membrane oxygenation in a patient with diffuse alveolar hemorrhage. Chest 2004; 126(1):305-309.

23. Jayne DR, Chapel H, Adu D, et al: Intravenous immunoglobulin for ANCA-associated systemic vasculitis with persistent disease activity. Q J Med 2000; 93: 433-439.

24. Pagnoux C, Mahr A, Hamidou MA, et al. Azathioprine or methotrexate maintenance for ANCA-associated vasculitis. N Engl J Med 2008; 359:2790-2803.

25. McCabe C, Jones Q, Nikolopoulou A et al. Pulmonary-renal syndromes: An update for respiratory physicians. Respiratory Medicine 2001; 105(10):1413-1421.

26. Betensley AD, Yankaskas JR. Factor viia for alveolar hemorrhage in microscopic polyangiitis. Am J Respir Crit Care Med 2002; 166(9):1291-2.

27. Santos -Ocampo AS,Mandell BF, Fessler BJ. Alveolar hemorrhage in systemic lupus erythematosus: presentation and management. Chest 2000; 118:1083-1090.

28. Claudio CP, Charbonney E, Durand M et al. Extracorporeal membrane oxygenation in diffuse alveolar hemorrhage secondary to systemic lupus erythematosus. J Clin Med Res 2014; 6(2): 145-148.

29. Levy JB, Turner AN, Rees AJ, Pusey CD. Long-term outcome of anti-glomerular basement membrane antibody disease treated with plasma exchange and immunosuppression Ann Intern Med 2001; 134(11):1033-42.

30. Deane K, West S. Antiphospholipid antibodies as a cause of pulmonary capillaritis and diffuse alveolar hemorrhage: a case series and literature review. Semin Arthritis and Rheumatism 2005; 35(3):154-165. 
31. Wanko S, Broadwater G, Folz R, Chao N. Diffuse Alveolar Hemorrhage: Retrospective review of clinical outcome in allogeneic transplant recipients treated with aminocaproic acid. Biol Blood Marrow Transplant 2006; 12(9):949-53.

32. de Prost N, Parrot A et al. Diffuse alveolar hemorrhage in immunocompetent patients: Etiologies and prognosis revisited. Respiratory medicine 2012; 106:1021-1032.

33. Lin Y, Zheng W, Tian X et al. Antineutrophil cytoplasmic antibody-associated vasculitis complicated with diffuse alveolar hemorrhage: a study of 12 cases. J Clin Rheumatol 2009; 15:341-344.

34. Gallagher H, Kwan J, Jayne D. Pulmonary renal syndrome: a 4-year, single-center experience. Am J Kidney Dis 2002; 39:42-47.

35. Martinez-Martínez MU, Abud-Mendoza C. Predictors of mortality in diffuse alveolar haemorrhage associated with systemic lupus erythematosus. Lupus 2011; 20 (6) 568-574.

36. Majhail NS, Parks K, Defor TE, et al. Diffuse alveolar hemorrhage and infection-associated alveolar hemorrhage following hematopoietic stem cell transplantation: related and highrisk clinical syndromes. Biol Blood Marrow Transplant 2006; 12:1038-1046. 